Article

\title{
Photocurable Bioinks for the 3D Pharming of Combination Therapies
}

\author{
Giovanny F. Acosta-Vélez ${ }^{1}\left(\mathbb{D}\right.$, Chase S. Linsley ${ }^{1} \mathbb{C}$, Timothy Z. Zhu ${ }^{1}$, Willie Wu ${ }^{1}$ \\ and Benjamin M. Wu ${ }^{1,2, *}$ \\ 1 Department of Bioengineering, University of California, Los Angeles, 420 Westwood Plaza, Room 5121, \\ Engineering V, 951600 Los Angeles, CA 90095, USA; gacosta3@g.ucla.edu (G.F.A.-V.); \\ clinsle@g.ucla.edu (C.S.L.); timmy90109@gmail.com (T.Z.Z.); williewu@ucla.edu (W.W.) \\ 2 Division of Advanced Prosthodontics and the Weintraub Center for Reconstructive Biotechnology, \\ University of California, 951600 Los Angeles, CA 90095, USA \\ * Correspondence: benwu@g.ucla.edu; Tel.: +1-(310)825-6215
}

Received: 11 November 2018; Accepted: 10 December 2018; Published: 11 December 2018

\begin{abstract}
Combination therapies mediate drug synergy to improve treatment efficacy and convenience, leading to higher levels of compliance. However, there are challenges with their manufacturing as well as reduced flexibility in dosing options. This study reports on the design and characterization of a polypill fabricated through the combination of material jetting and binder jetting for the treatment of hypertension. The drugs lisinopril and spironolactone were loaded into hydrophilic hyaluronic acid and hydrophobic poly(ethylene glycol) (PEG) photocurable bioinks, respectively, and dispensed through a piezoelectric nozzle onto a blank preform tablet composed of two attachable compartments fabricated via binder jetting 3D printing. The bioinks were photopolymerized and their mechanical properties were assessed via Instron testing. Scanning electron microscopy (SEM) was performed to indicate morphological analysis. The polypill was ensembled and drug release analysis was performed. Droplet formation of bioinks loaded with hydrophilic and hydrophobic active pharmaceutical ingredients (APIs) was achieved and subsequently polymerized after a controlled dosage was dispensed onto preform tablet compartments. High-performance liquid chromatography (HPLC) analysis showed sustained release profiles for each of the loaded compounds. This study confirms the potential of material jetting in conjunction with binder jetting techniques (powder-bed 3D printing), for the production of combination therapy oral dosage forms involving both hydrophilic and hydrophobic drugs.
\end{abstract}

Keywords: 3D printing; pharmaceutical tablets; poly(ethylene glycol); hyaluronic acid; photopolymerization; inkjet printing

\section{Introduction}

Personalized medicine is becoming a reality. For instance, most hearing aids are now custom-fit to each user's ear canal [1] and the manufacturing process for Invisalign ${ }^{\mathrm{TM}}$ aligners and retainers utilizes 3D printing [2]. Additionally, custom bioresorbable tracheal splints fabricated using laser-based 3D printing were successfully used to treat children with tracheobronchomalacia [3]. Recently, interest in applying 3D printing to the manufacturing of pharmaceutical tablets—known as 3D pharming [4] —has been gaining traction since it can allow for on-demand manufacturing of personalized pharmaceutical dosage forms [5,6]. It also allows for the creation of dosage forms with increased complexity, including the manufacturing of oral dosages containing multiple active pharmaceutical ingredients (APIs), which aim to enhance patient compliance by reducing the number of pills required on a daily basis [7]. 
Additionally, several medical conditions utilize combination therapies to improve treatment efficacy, such as hypertension, HIV infection, depression, type 2 diabetes, and cancer [8-17].

There are several challenges to manufacturing dosage forms containing multiple APIs as well as dosing constraints that impact treatment efficacy. Currently, a few fixed dose combinations of varying strength are created for any given combination therapy. However, patients often require dose adjustments to the extent that a tablet with multiple APIs is not a suitable form factor [18]. Additionally, there are challenges to manufacturing multiple-API dosage forms [19]. For instance, a common challenge is designing a single dosage form that combines two chemically incompatible APIs that have to be kept separated. This can result in higher manufacturing costs since a more complex dosage form has to be produced. Three-dimensional pharming is uniquely positioned to overcome these obstacles. In fact, techniques such as fused deposition modeling [20] and extrusion printing at room temperature $[21,22]$ have demonstrated feasibility in the manufacturing of pharmaceutical tablets with multiple APIs. Material jetting technologies, however, allow for compositional control at a voxel level, thereby enabling precise dosage control that is tailorable to each individual patient. Additionally, material can be dispensed at room temperature, which is compatible with thermally labile APIs.

The focus of this work was to demonstrate that 3D printing and the development of photocurable formulations can be combined to fabricate pharmaceutical tablets with controlled dosages of both hydrophilic and hydrophobic APIs, and act as a potential carrier for personalized medicine treatments. Previously, this lab engineered a hyaluronic acid-based photocurable bioink for the 3D pharming of hydrophilic compounds via material jetting [23]. Additionally, this lab engineered a poly(ethylene glycol) diacrylate-based bioink capable of loading hydrophobic drugs [24]. In this paper, both bioinks are used to manufacture a tablet with multiple APIs used to treat hypertension. Specifically, the APIs lisinopril and spironolactone were chosen as model drugs for this combination therapy. Lisinopril, an angiotensin-converting enzyme inhibitor (ACEI) for the treatment of hypertension [25,26], was chosen due to its hydrophilicity, and spironolactone, a potassium-sparing diuretic for the treatment of hypertension and congestive heart failure [27], was selected because of its hydrophobicity. The formulated bioinks were dispensed through a piezoelectric nozzle at room temperature into a blank preform tablet featuring two compartments, one for each formulation. Compartmentalizing the preform tablet was required in order to expose each formulation, separately, to the required light exposure times resulting in optimal mechanical properties and release profiles. The preform tablet parts were manufactured by binder jetting process, using calcium sulfate powder as the excipient. The drug-loaded bioinks were subsequently photopolymerized, and the preform tablet parts were assembled to finalize the pharmaceutical dosage form. This tablet produced a sustained drug release profile for both APIs and demonstrated that this 3D pharming approach could successfully fabricate oral combination therapies at room temperature, with quick manufacturing times and controlled dosages.

\section{Materials and Methods}

\subsection{Hydrophilic Photocurable Bioink Preparation}

Hyaluronic acid norbornene (HANB) was synthesized as previously described [23]. Briefly, hyaluronic acid (HA) (60 kDa.MW) (Genzyme Corporation, Cambridge, MA, USA) was modified with hydrazide groups through a reaction with adipic acid dihydrazide (ADH) in the presence of 1-ethyl-3-(dimethylaminopropyl) carbodiimide hydrochloride (EDC). The product was dialyzed for 3 days against deionized water (DI) water (Fisherbrand regenerated cellulose, MWCO 12,000-14,000 Da, Houston, TX, USA), frozen, and lyophilized. On a second reaction, the HA functionalized with hydrazide groups (HA-ADH) was reacted cis-5-norbornene-endo-2,3-dicarboxylic anhydride (Sigma-Aldrich, St. Louis, MO, USA), resulting in norbornene-functionalized HA. The product was dialyzed against DI water for 3 days, filtered, lyophilized, and stored at $-20{ }^{\circ} \mathrm{C}$. 
Norbornene-functionalized HA was characterized by proton nuclear magnetic resonance spectroscopy $\left({ }^{1} \mathrm{H}\right.$ NMR) on a Bruker AV300 broad band FT NMR Spectrometer (Billerica, MA, USA). The degree of modification obtained was $\sim 50 \%$.

Following its synthesis, HANB was dissolved in PBS and mixed with poly(ethylene glycol) dithiol (1500 Da, PEGDT) at a crosslinking ratio (ratio of thiol groups to norbornene groups, $r_{\text {ratio }}$ ) of 0.6 . Norbornene-functionalized HA was added at a weight percent $\left(W_{\text {HANB }}\right)$ of $3 \%$. Eosin $Y$ was added as a photoinitiator and poly(ethylene glycol) (PEG, $200 \mathrm{Da}$ ) was added to optimize the viscosity of the formulation for droplet formation. Each of these two components constituted $10 \% \mathrm{v} / \mathrm{v}$ of the bioink. Lisinopril dihydrate (Fisher Scientific, Pittsburgh, PA, USA) was added at a concentration of $40 \mathrm{mg} / \mathrm{mL}$. All chemicals were purchased from Sigma-Aldrich unless otherwise stated.

\subsection{Hydrophobic Photocurable Bioink Preparation}

The hydrophobic bioink was formulated as previously described [24], with minor modifications. Briefly, the bioink was composed of 30\% poly(ethylene glycol) diacrylate (PEGDA, $250 \mathrm{Da}$ ), 50\% PEG200, and 20\% ethanol. Eosin Y (1.0 mM) and mPEG-amine (0.05 M) (350 Da, Creative PEG Works, Chapel Hill, NC, USA) were added as photoinitiator and co-initiator, respectively. Spironolactone was added at a concentration of $20 \mathrm{mg} / \mathrm{mL}$. All chemicals were purchased from Sigma-Aldrich unless otherwise stated.

\subsection{Bioinks Gelation and Mechanical Properties}

The tensile strength of polymerized gels was analyzed to characterize the mechanical properties of the bioinks. One-mL syringes (BD \& Co., Franklin Lakes, NJ, USA), modified by eliminating their tips, were loaded with $50 \mu \mathrm{L}$ of bioink. Gels were formed with hydrophilic and hydrophobic bioinks by exposing them to visible light at an intensity of $120 \mathrm{~mW} / \mathrm{cm}^{2}$ for a period of $2 \mathrm{~min}$ and $1 \mathrm{~min}$, respectively. Additionally, the tensile strength of gels with varied drug concentrations was measured to analyze the impact of drug load on the mechanical properties of the polymerized bioinks. An Instron (5564 model) was used to measure the failure load of the gels fabricated. The tensile strength $(\sigma)$ was calculated through Equation (1), where $D$ is the gel diameter, $H$ is the thickness, and $F$ represents the failure load [28].

$$
\sigma=\frac{2 F}{\pi D H}
$$

The inverse of the Ohnesorge number ( $Z$ value) of the bioinks was calculated to assess the printability of these solutions through inkjet printing piezoelectric nozzles. Equation (2) defines the $Z$ value, where $a$ is the radius of the piezoelectric nozzle printing orifice and $\rho, \gamma$, and $\eta$ represent the density, surface tension, and viscosity of the photocurable formula, respectively [29].

$$
Z=\frac{(a \rho \gamma)^{1 / 2}}{\eta}
$$

The surface tension was measured with a tensiometer (Kimble Chase 14,818 Tensiometer, Cole-Parmer, Vernon Hills, IL, United States) and calculated by using Equation (3), where $h$ is the distance between menisci of the formulation in the test tube and the one in the capillary tube, $r$ is the radius of the capillary, $\rho$ is the density of the formulation, and $g$ is the acceleration due to gravity [30]. One $\mathrm{mL}$ of each bioink was weighted and the mass obtained was divided by the pre-determined volume to calculate the density. The viscosity of the bioinks was measured with a rheometer (Discovery HR-2, TA Instruments, New Castle, DE, USA). A cone and plate geometry (using a 40-mm 2.016 ${ }^{\circ}$ was utilized for the experiment, with a shear rate ranging from 10 to $100 \mathrm{~Hz}$.

$$
\gamma=\frac{1}{2} h r \rho g
$$




\subsection{Scanning Electron Microscopy (SEM)}

The morphology of the gels was observed with a NOVA 230 NanoSEM scanning electron microscope. Images of lyophilized hydrophilic gels were taken, as well as cross-sectional images of hydrophobic gels.

\subsection{Preform Tablet Fabrication and Characterization}

The drug-containing bioinks were directly printed into tablet preforms that were fabricated by 3D printing (ProJet 660; 3D Systems, Inc.; Rock Hill, SC, USA). The printing materials consisted of calcium sulfate hemihydrate powder (VisiJet PXL Core; 3D Systems, Inc.; Rock Hill, SC, USA), and a liquid inkjet binder comprised of deionized water containing $5 \%$ ethanol and $0.25 \%$ Tween 80 . The polypill preform tablet was designed with two separate chambers that could each hold up to $250 \mu \mathrm{L}$ of ink and be assembled into a single tablet. The assembled dimensions were kept below the 22-mm maximum tablet size recommended by the Food and Drug Administration (FDA) [31]. Additionally, each chamber was independently modified to prevent absorption of the respective drug-containing bioink into the preform tablet during printing, as previously reported [23]. Briefly, the chamber holding the hydrophobic formulation was infused with PEG $(35 \mathrm{kDa})$ by submerging the tablet in an acetone solution containing $15 \%(\mathrm{w} / \mathrm{w})$ PEG for $30 \mathrm{~min}$ at $55^{\circ} \mathrm{C}$. Next, the well was brush-coated with Eudagrit ${ }^{\circledR}$ E100 (Evonik, Essen, Germany) (polymethacrylate copolymer) dissolved in acetone at 20\% $(\mathrm{w} / \mathrm{w})$. The chamber holding the hydrophilic formulation was infused with Eudagrit ${ }^{\circledR}$ E100 dissolved in acetone at $10 \%(\mathrm{w} / \mathrm{w})$. Surface morphology of the preform tablet was characterized by scanning electron microscopy (NOVA NanoSEM 230, FEI Co., Hillsboro, OR, USA).

\subsection{Drug Release Kinetics}

A piezoelectric dispenser with a nozzle diameter of $80 \mu \mathrm{m}$ (MJ-ABP-01-080, MicroFab, Plano, TX, USA) was used to assess the droplet formation capability of the engineered bioinks. This dispenser was controlled with a microdispensing system (MD-E-3000, Microdrop, Norderstedt, Germany). The dispensing parameters used were $46 \mathrm{~V}$, a 16- $\mu \mathrm{m}$ pulse width, and a frequency of $2000 \mathrm{~Hz}$. The droplet formation process was captured with an analog camera (JAI CV-S3300), equipped with a lens (Edmund Optics, Barrington, NJ, USA). A light-emitting diode (LED) was connected to the microdispensing system in order to control its strobe delay.

Lisinopril-loaded hydrophilic bioink was dispensed in the bottom part of the polypill $(250 \mu \mathrm{L})$ and exposed to visible light $\left(120 \mathrm{~mW} / \mathrm{cm}^{2}\right)$ for $2 \mathrm{~min}$, to induce gelation of the hydrogel precursor solution. Spironolactone-loaded hydrophobic bioink was dispensed into the upper piece of the polypill $(125 \mu \mathrm{L})$ and exposed to light for $1 \mathrm{~min}$. Following the gelation of the bioinks, the pieces of the polypill were assembled to finalize the pharmaceutical product.

The tablets were placed into uni-cassettes (Tissue-Tek) and immersed into beakers containing $500 \mathrm{~mL}$ of dissolution medium (monobasic potassium phosphate $1.053 \mathrm{mM}, \mathrm{pH}$ of 2.5), conditioned at $37^{\circ} \mathrm{C}$ and stirred at $60 \mathrm{rpm}$. Aliquots of $1 \mathrm{~mL}$ were taken after $0.5,2,4,6,8,12,18$, and $24 \mathrm{~h}$. The volume removed was replenished with fresh dissolution medium conditioned at $37^{\circ} \mathrm{C}$. The drug concentration in each aliquot was measured via HPLC (Waters 2690 with a PDA 996 detector). The wavelength used to detect the APIs were $220 \mathrm{~nm}$ and $240 \mathrm{~nm}$ for lisinopril and spironolactone, respectively.

\subsection{Statistical Analysis}

Statistical analysis was performed with GraphPad Prism software (GraphPad Software, Inc., San Diego, CA, USA). Statistical significance was assessed using single factor ANOVA test with a Tukey post-test and $95 \%$ confidence interval. 


\section{Results and Discussion}

\subsection{Bioinks Characterization}

Hyaluronic acid is a natural glycosaminoglycan found in connective, neural, and epithelial tissues [32,33]. The biocompatibility of hyaluronic acid hydrogels has been assessed in tissue engineering studies, where diverse cell types have been cultured in hydrogels for the formation of tissues and the study of biological processes [34-36]. Moreover, hyaluronic acid photocurable formulations can polymerize under quick gelation times (Figure S1). Lisinopril was dissolved in a hyaluronic acid-based hydrophilic bioink at a concentration of $40 \mathrm{mg} / \mathrm{mL}$. The lisinopril formulation was dispensed into a modified syringe $(50 \mu \mathrm{L})$ and exposed to visible light at an intensity of $120 \mathrm{~mW} / \mathrm{cm}^{2}$ for $2 \mathrm{~min}$ (Figure 1). The storage modulus of the resulting hydrogel was quantified to assess the mechanical properties of the polymerized bioink, due to its viscoelastic property. Additionally, hydrogels with different drug concentrations (20 and $10 \mathrm{mg} / \mathrm{mL}$ ) were polymerized to study the effect of drug load on the mechanical properties of this hydrophilic material (Figure 2A). The results indicate that this hydrophilic material has a low storage modulus due to its elevated water content and the low $W_{\text {HANB }}(3 \%)$ utilized for its fabrication. The elevated water content of the hydrogel allows for the effective diffusion of the hydrophilic API out of the oral dosage form, given the dissolution of the preform tablet designed to disintegrate under acidic conditions similar to the ones found in the stomach. The hydrogels had an average $\mathrm{G}^{\prime}$ of $1003.86 \mathrm{~Pa}$. Figure 2A indicates that drug load had no influence in the mechanical properties of the hydrogel, where larger drug concentrations had no impact over the $G^{\prime}$ of the gels. This result is consonant with the $G^{\prime}$ data previously shown [23], where ropinirole-loaded at a concentration of $40 \mathrm{mg} / \mathrm{mL}$ had no impact on the mechanical properties of the gel, compared to hydrogels with no drug. However, a decrease in $\mathrm{G}^{\prime}$ was noticed on hydrogels loaded with ropinirole at a concentration of $80 \mathrm{mg} / \mathrm{mL}$. It can be stated that the $\mathrm{G}^{\prime}$ remains stable for this lisinopril formulation at concentrations between 0 and $40 \mathrm{mg} / \mathrm{mL}$, the maximum lisinopril concentration achievable in the hyaluronic acid solution.

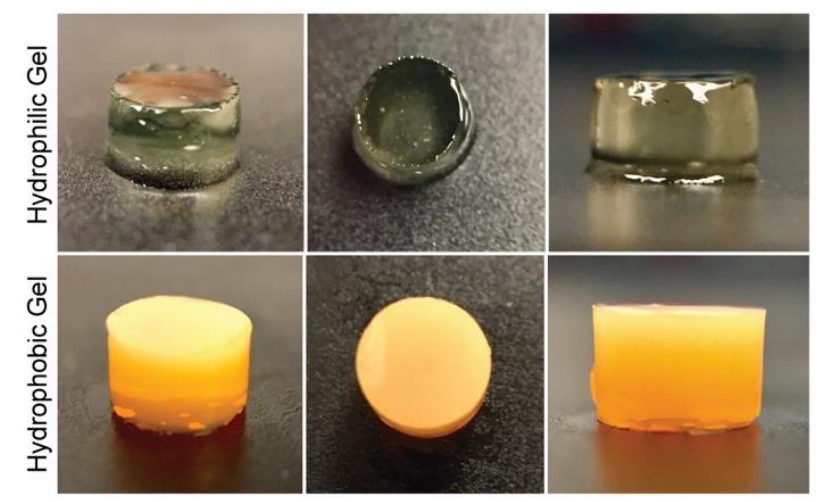

Figure 1. Polymerized drug-loaded bioinks. The upper row shows images of the polymerized hyaluronic acid-based bioink, loaded with lisinopril at a concentration of $40 \mathrm{mg} / \mathrm{mL}$. This bioink has a $W_{\text {HANB }}$ value of $3 \%$ and a $T_{\mathrm{L}}$ of $2 \mathrm{~min}$. The bottom images show polymerized poly(ethylene

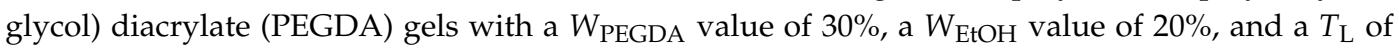
$1.0 \mathrm{~min}$.

Spironolactone was chosen as the second model drug in this study due to its high hydrophobicity. The model drug was dissolved in a PEG-based bioink specifically engineered for hydrophobic drugs, at a concentration of $20 \mathrm{mg} / \mathrm{mL}$. Fifty $\mu \mathrm{L}$ of formulation were pipetted into a modified syringe, the solution was exposed to light for 1 min (Figure 1), and the tensile strength of the resulting gel was measured (Figure 2B). Results show an average tensile strength for this material of $176.66 \mathrm{kPa}$. The typical tensile strength of pharmaceutical tablets is within the range of 1 and $10 \mathrm{MPa}$ [37]. The $\mathrm{G}^{\prime}$ obtained for this gel is well below this range, and therefore, this material should be used 
in combination with a preform tablet that provides support and physical stability to the oral dosage form. Furthermore, the effect of drug concentration on the mechanical properties of the gel was studied by measuring the tensile strength of gels containing $100,80,40$, and $20 \mathrm{mg} / \mathrm{mL}$. Figure $2 \mathrm{~B}$ shows that drug concentration had no impact on the tensile strength of the material. This contrasts with results previously demonstrated [24], where a decrease in tensile strength was observed with increasing naproxen and ibuprofen concentrations. This divergence is due to the lower $W_{\text {PEGDA }}$ value used in this formulation (30\%). These results indicate that softer materials consisting of photo-polymerized bioinks, such as the hyaluronic acid hydrogel, tend to have a steady mechanical stability when exposed to increasing drug concentrations. Stronger gels can experience substantial differences in mechanical properties (Figure S2).

A.

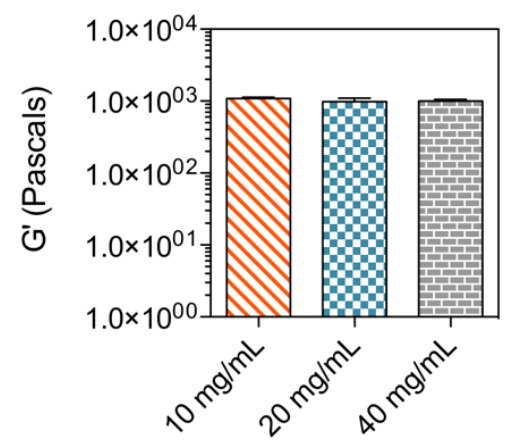

B.

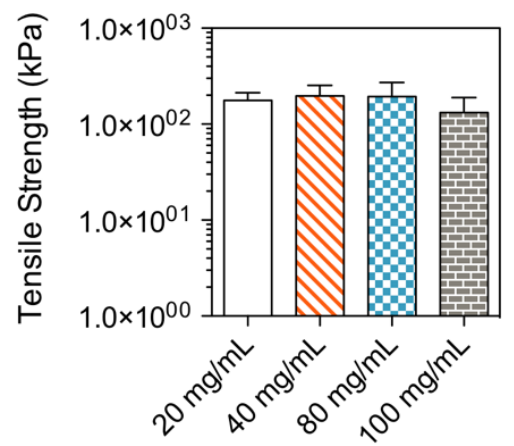

Figure 2. Tensile strength of lisinopril and spironolactone gels. (A) Tensile strength of lisinopril tablets with diverse drug concentrations. Forty $\mathrm{mg} / \mathrm{mL}$ was the maximum solubility achieved for this active pharmaceutical ingredient (API) in this bioink formulation. No statistical difference was observed between samples with varying lisinopril concentrations. (B) Tensile strength of spironolactone tablets with diverse drug concentrations. One-hundred $\mathrm{mg} / \mathrm{mL}$ was the maximum solubility achieved for this API. No statistical difference was observed between samples with varying concentrations.

Scanning electron microscopy imaging was performed on both gels to observe the morphology of the polymerized structures. Figure 3 shows an irregular surface in both gels with small crevices that further facilitate the release of drugs, that otherwise diffuse out of the gels through their polymerized chemical structures. The wrinkles observed in the hydrophilic gel (Figure 3A) are a consequence of the lyophilized/dehydrated nature of the sample tested.

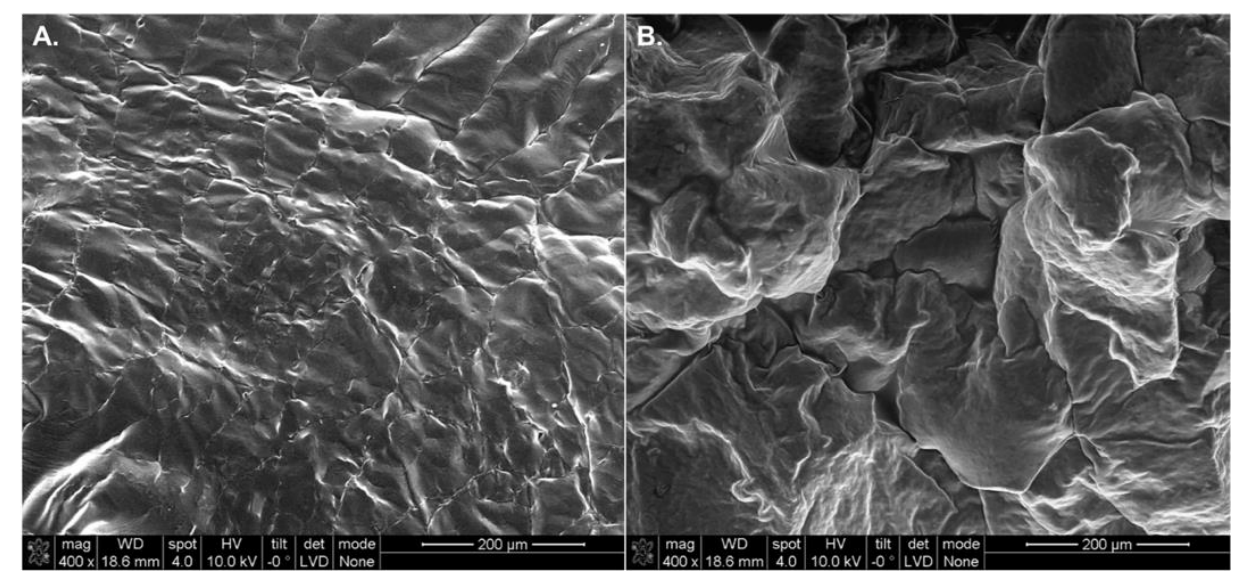

Figure 3. Cross-sectional SEM micrographs of polymerized bioinks loaded with lisinopril and spironolactone. (A) Lyophilized lisinopril-loaded hydrogel $\left(40 \mathrm{mg} / \mathrm{mL}\right.$ ) with a $W_{\text {HANB }}$ of $3 \%$ and a $r_{\text {ratio }}$ of 0.6. This bioink was exposed to a $T_{\mathrm{L}}$ of $2 \mathrm{~min}$. (B) Polymerized hydrophobic ink loaded with spironolactone $(20 \mathrm{mg} / \mathrm{mL})$. This formulation contained a $W_{\text {PEGDA }}$ of $20 \%$ and a $T_{\mathrm{L}}$ of $1 \mathrm{~min}$. 


\subsection{Droplet Formation}

To study the droplet formation ability of these bioinks, the inverse of the Ohnesorge number, denominated as $Z$ value, was calculated (Equation (3)). This dimensionless number considers the inertia and surface tension forces of a fluid over its viscosity forces to define its droplet formation ability. The orifice radius of the nozzle used for the inkjet printing of the fluid is also a factor taken into consideration within this number. $Z$ values between 4 and 14 are considered printable fluids [29]. Values above 14 typically exhibit the formation of satellite droplets, whereas values below four present strong viscous forces. The viscosity, surface tension, and density of the bioinks was quantified, in order to determine their $Z$ value. Table 1 shows the results obtained for these parameters at room temperature and the $Z$ value for the two bioinks utilized in this study. The hydrophilic bioink experienced a higher viscosity value $(9.83 \mathrm{cP})$, resulting in a lower $Z$ value than the hydrophobic formulation. The later one had lower viscosity and surface tension parameters $(4.88 \mathrm{cP}$ and $31.41 \mathrm{mN} / \mathrm{m}$, respectively), resulting in a higher $Z$ value of 10.52 . However, both formulations fell within the defined range for printable fluids as depicted in Figures 4 and 5, where the droplet formation sequence of these bioinks can be observed.

Table 1. Physical properties and $Z$ value of formulated bioinks.

\begin{tabular}{|c|c|c|c|c|c|}
\hline Bioink & $r$ & $P\left(\mathrm{~kg} / \mathrm{m}^{3}\right)$ & $\gamma$ & $\eta(\mathrm{mPa} \cdot \mathrm{s})$ & $Z$ \\
\hline Hydrophilic & 0.08 & 1022.27 & 57.76 & 9.83 & 6.99 \\
\hline Hydrophobic & 0.08 & 1048.00 & 31.41 & 4.88 & 10.52 \\
\hline
\end{tabular}

Figure 4. Droplet formation sequence for hyaluronic acid-based bioink with a $W_{\text {PEGDA }}$ of $3 \%$, a $r_{\text {ratio }}$ of 0.6 , and loaded with lisinopril at a concentration of $40 \mathrm{mg} / \mathrm{mL}$. This formulation had a $Z$ value of 6.99 , falling within the printable range (4-14).

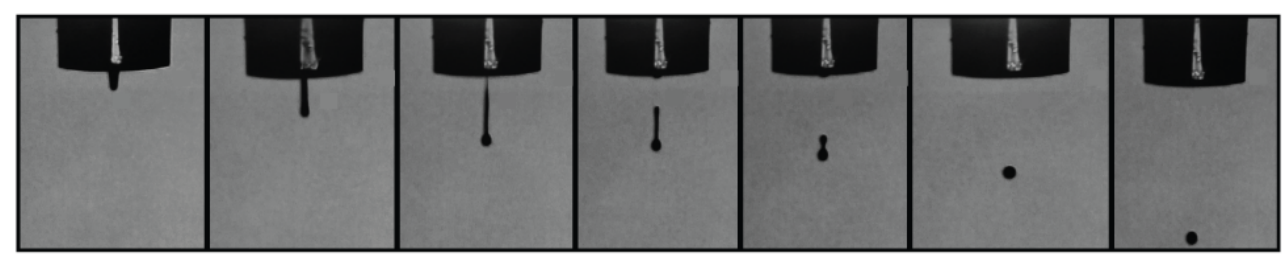

Figure 5. Droplet formation sequence for PEGDA-based bioink with a $W_{\text {PEGDA }}$ of $30 \%$ and loaded with spironolactone at a concentration of $20 \mathrm{mg} / \mathrm{mL}$. This formulation had a $Z$ value of 10.52 , falling within the printable range (4-14).

\subsection{Preform Tablet Characterization}

The role of the preform tablet was to serve as a vessel for the formulations dispensed prior to their curing, given that their polymerization occurred after a desired amount of formulation was allotted. There was a need to compartmentalize the tablet for each formulation added, since each formulation was cured for different light exposure times to obtain the desired mechanical properties and drug release profiles. Calcium sulfate hemihydrate was clinically used in the preparation of plaster of Paris, which is used for casts that immobilize fractures, and is not used in tablet formulations [38]. Calcium sulfate dihydrate, however, has been commonly used in pharmaceutical applications [39], and the dihydrate is formed when hemihydrate is mixed with water [40]. In this study, water was used as the liquid binder during the fabrication of the perform tablets via 3D printing (Figure 6). Upon contact with 
the powder, water causes the dissolution of the calcium sulfate hemihydrate and recrystallization of the dihydrate form [41]. The SEM micrographs (Figure 7A) show that pores exist within the uncoated preform tablet wall. These pores negatively impacted the performance of tablets during printing of the bioink. Specifically, the porosity in the preform tablet allowed both the hydrophilic and hydrophobic bioinks to soak into the preform tablet during printing, which not only weakened the preform tablet, but also negates the advantages of using 3D printing for pharmaceutical applications, such as control over drug positioning. To fill the pores, two different coatings were used depending on the ink being used for printing. For the hydrophilic bioink, the preform tablets were soaked in Eudagrit ${ }^{\circledR E} 100$ (Figure 7B). For the hydrophobic bioink, the preform tablets were first soaked in a high-molecular weight PEG (35 kDa) solution. High-molecular weight PEG was selected because it is immiscible with the low-molecular weight PEGDA in the bioink (Figure 7C). Additionally, a thin coating of Eudagrit ${ }^{\circ} 100$ was added to the preform tablet well to further inhibit the absorption of the bioink during printing. The SEM micrographs show that the polymeric coating fills the pores of the preform tablet (Figure 7D).

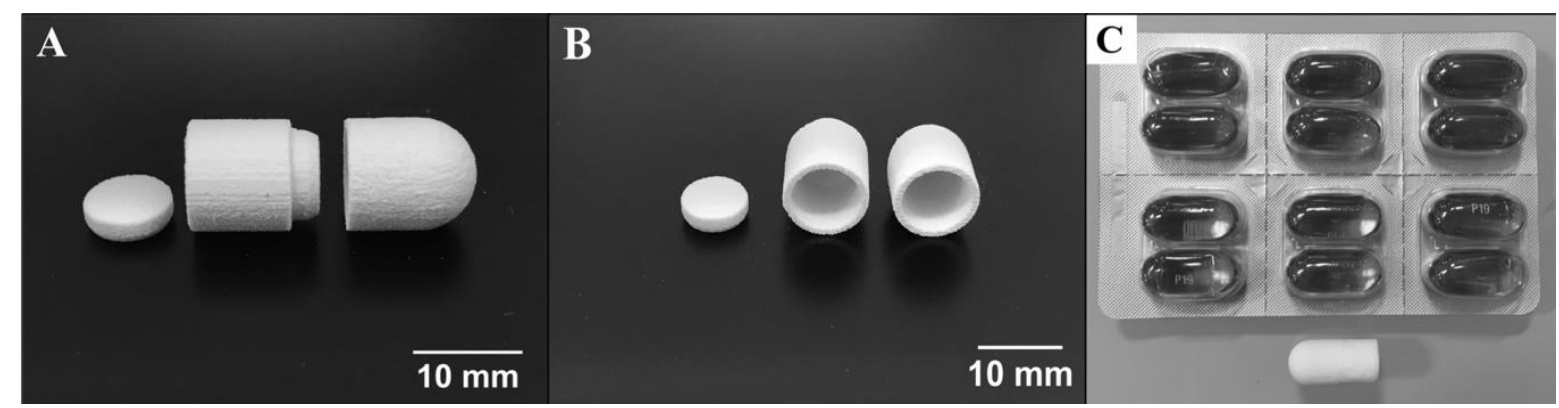

Figure 6. Multi-compartment preform tablet. (A) Side view of the three pieces constituting the preform tablet. From left to right, top cap, top compartment, and bottom compartment. These pieces were manufactured by powder-bed 3D printing using as binder DI water with $5 \%$ ethanol and $0.25 \%$ Tween 80. The powder utilized for their construction was calcium sulfate. Each of the two wells have a $250 \mu \mathrm{L}$ capacity. (B) Front view of the preform tablet fabricated. (C) Comparison between the assembled version of the preform tablet and commercially available gel capsules.
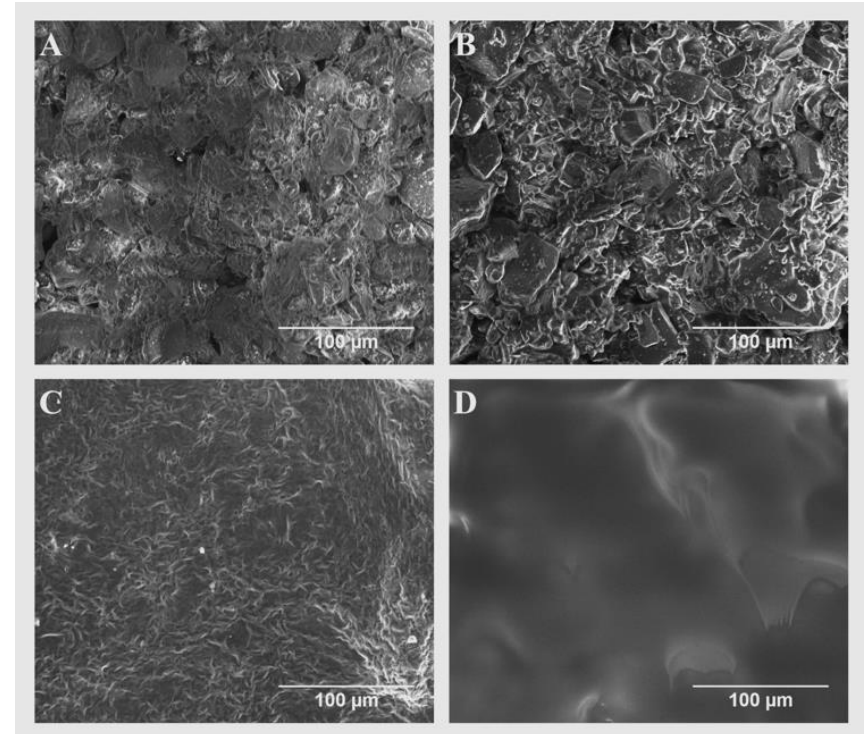

Figure 7. The SEM micrographs of the untreated and coated preform tablet surfaces. (A) Surface of untreated preform tablet. (B) Bottom compartment infused with a 10\% E-100 in acetone solution. (C) Top compartment infused with a 15\% PEG (35 kDa) in acetone solution. (D) Inner section of the top compartment preform tablet, brushed with a $20 \%$ E-100 in acetone solution. 


\subsection{Polypill Dissolution Test}

The hyaluronic acid-based hydrophilic bioink was loaded with lisinopril at a concentration of $40 \mathrm{mg} / \mathrm{mL}$. Two-hundred-and-fifty $\mu \mathrm{L}$ of this formulation were dispensed into the bottom compartment of the preform tablet and further exposed to visible light for $2 \mathrm{~min}$, to induce gelation of the photocurable bioink. Likewise, the PEG-based hydrophobic bioink was loaded with spironolactone at a concentration of $20 \mathrm{mg} / \mathrm{mL}$. One-hundred-and-twenty-five $\mu \mathrm{L}$ of this formulation were loaded into the top compartment of the preform tablet and exposed to visible light for a period of $1 \mathrm{~min}$ (Figure 8). Once the bioinks were polymerized, the two compartments were attached, and the small cap was placed to seal the top compartment, completing the oral dosage form. The tablet was immersed in a beaker containing $500 \mathrm{~mL}$ of dissolution medium, conditioned at $37^{\circ} \mathrm{C}$ and stirred at $60 \mathrm{rpm}$. Aliquots were taken after $0.5,2,4,6,8,12,18$, and $24 \mathrm{~h}$ of dissolution and their drug concentration was assessed through HPLC analysis. The results obtained show a dual sustained release of lisinopril and spironolactone in a period of $24 \mathrm{~h}$ (Figure 9). The preform tablet dissolved almost in its entirety with the exception of the lower part of the top compartment, completely exposing the gels to the dissolution medium (Figure S3). Lisinopril experienced faster drug release kinetics, when compared to spironolactone. This result can be explained by the differences in the microarchitecture and composition of the gels. The hydrophilic formula has over $90 \%$ of water content, facilitating the diffusion of lisinopril, a hydrophilic compound, into the dissolution medium. Moreover, the hydrogel has a $W_{\text {HANB }}$ of only $3 \%$, allowing small molecules to easily diffuse through the polymerize matrix. The hydrophobic formulation has a higher polymeric content $\left(W_{\mathrm{PEGDA}}=30 \%\right)$ resulting in a mesh size of $\sim 11 \AA$ [24]. Naproxen and ibuprofen have a hydrodynamic radius of $3.77 \AA$ and $6.80 \AA$ [42], respectively, and molecular weights of $230.26 \mathrm{Da}$ and $206.29 \mathrm{Da}$. It can be hypothesized that the hydrodynamic radius of spironolactone is close to the mesh size of the polymerized hydrophobic gel $(11 \AA)$, since it has a significantly higher molecular weight than naproxen and ibuprofen, model drugs previously utilized under similar experiment conditions [24]. This would explain the slower release profile observed with spironolactone, since diffusion of the molecule through the gel matrix would be impeded. The use of a higher PEGDA molecular weight for the fabrication of the gel could result in larger mesh sizes and consequently, enhanced drug release kinetics.

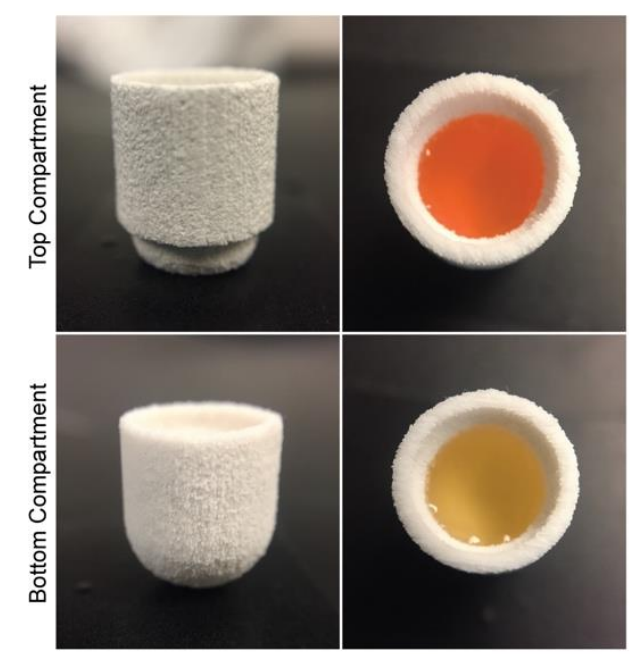

Figure 8. Multi-compartment preform tablet loaded with hydrophilic (bottom) and hydrophobic (top) bioinks.

The dual release of these APIs for the treatment of hypertension demonstrates the use of inkjet printing for the fabrication of combination therapies. Moreover, it shows that the therapy could contain both hydrophilic and hydrophobic compounds. This technology would be especially applicable towards drugs that achieve their pharmacological effect at low dosages and could be targeted towards 
the development of oral dosage forms for children who require small dosages not always commercially available. Moreover, children experience drastic changes in metabolism that affect the dosage needed to achieve a given target pharmacological effect.

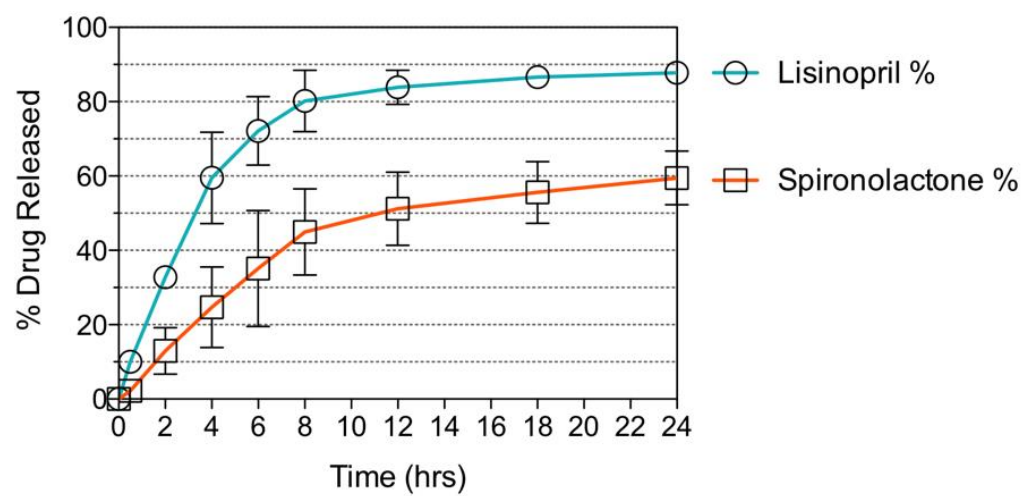

Figure 9. Dissolution test of polypill. A sustained release profile was achieved for both APIs. Around $90 \%$ of the lisinopril loaded was release in a period of $24 \mathrm{~h}$, releasing most of the drug within the first $8 \mathrm{~h}$. Above $60 \%$ of the spironolactone loaded was released in a period of $24 \mathrm{~h}$.

\section{Conclusions}

In this study, a combination therapy oral dosage form for the treatment of hypertension was designed. The polypill featured the use of a hyaluronic acid photocurable hydrophilic bioink and a PEG photocurable hydrophobic bioink, loaded with lisinopril and spironolactone, respectively. A preform tablet with two compartments able to hold these bioinks was designed and manufactured through binder jetting 3D printing. The formulations were dispensed through a piezoelectric nozzle designed for material jetting and subsequently polymerized through exposure to visible light. The preform parts were assembled and a dissolution study was carried out, where a dual sustained release of the drugs lisinopril and spironolactone was observed over a period of $24 \mathrm{~h}$. This study shows the feasibility of 3D printing photocurable formulations to manufacture combination therapy oral dosage forms that incorporate both hydrophilic and hydrophobic APIs, with a special application towards drugs that achieve their pharmacological effect at low dosages.

Supplementary Materials: The following are available online at http:/ / www.mdpi.com/2073-4360/10/12/1372/ s1, Figure S1: Gelation kinetics obtained through in situ photorheology of the hydrophilic bioink, Figure S2: Tensile Strength of PEGDA-based $\left(W_{\text {PEGDA }}=100 \%\right)$ gels loaded with varying concentrations of spironolactone, Figure S3: Exposed gels after $24 \mathrm{~h}$ of dissolution time.

Author Contributions: Conceptualization, B.W., G.A., and C.L.; Investigation, G.A., C.L., T.Z., and W.W.; Resources, B.W.; Data curation, G.A., C.L.; Writing—original draft preparation, G.A.; Writing-review and editing, C.L.; Funding acquisition, B.W.

Funding: This research was funded by GlaxoSmithKline, grant number PO No. 36,257,529 OS.

Conflicts of Interest: The authors declare no conflict of interest.

\section{References}

1. Banks, J. Adding value in additive manufacturing: Researchers in the United Kingdom and Europe look to 3D printing for customization. IEEE Pulse 2013, 4, 22-26. [CrossRef] [PubMed]

2. Kaye, R.; Goldstein, T.; Zeltsman, D.; Grande, D.A.; Smith, L.P. Three dimensional printing: A review on the utility within medicine and otolaryngology. Int. J. Pediatr. Otorhinolaryngol. 2016, 89, 145-148. [CrossRef] [PubMed]

3. Zopf, D.A.; Hollister, S.J.; Nelson, M.E.; Ohye, R.G.; Green, G.E. Bioresorbable Airway Splint Created with a Three-Dimensional Printer. N. Engl. J. Med. 2013, 368, 2043-2045. [CrossRef] [PubMed]

4. Acosta-Vélez, G.F.; Wu, B.M. 3D Pharming: Direct Printing of Personalized Pharmaceutical Tablets Abstract Powder Bed Inkjet 3D Printing. Polym. Sci. 2016, 1, 1-10. 
5. Norman, J.; Madurawe, R.D.; Moore, C.M.V.; Khan, M.A.; Khairuzzaman, A. A new chapter in pharmaceutical manufacturing: 3D-printed drug products. Adv. Drug Deliv. Rev. 2017, 108, 39-50. [CrossRef] [PubMed]

6. Boudriau, S.; Hanzel, C.; Massicotte, J.; Sayegh, L.; Wang, J.; Lefebvre, M. Randomized Comparative Bioavailability of a Novel Three-Dimensional Printed Fast-Melt Formulation of Levetiracetam Following the Administration of a Single 1000-mg Dose to Healthy Human Volunteers Under Fasting and Fed Conditions. Drugs R D 2016, 16, 229-238. [CrossRef] [PubMed]

7. Preis, M.; Öblom, H. 3D-Printed Drugs for Children-Are We Ready Yet? AAPS PharmSciTech 2017, 18, 303-308. [CrossRef] [PubMed]

8. Chow, C.K.; Thakkar, J.; Bennett, A.; Hillis, G.; Burke, M.; Usherwood, T.; Vo, K.; Rogers, K.; Atkins, E.; Webster, R.; et al. Quarter-dose quadruple combination therapy for initial treatment of hypertension: Placebo-controlled, crossover, randomised trial and systematic review. Lancet 2017, 389, 1035-1042. [CrossRef]

9. Weichselbaum, R.R.; Liang, H.; Deng, L.; Fu, Y.-X. Radiotherapy and immunotherapy: A beneficial liaison? Nat. Rev. Clin. Oncol. 2017, 14, 365-379. [CrossRef]

10. Gradman, A.H.; Basile, J.N.; Carter, B.L.; Bakris, G.L. Combination therapy in hypertension. J. Am. Soc. Hypertens. 2016, 4, 42-50. [CrossRef]

11. Cahn, A.; Cefalu, W.T. Clinical considerations for use of initial combination therapy in type 2 diabetes. Diabetes Care 2016, 39, S137-S145. [CrossRef] [PubMed]

12. Melero, I.; Berman, D.M.; Aznar, M.A.; Korman, A.J.; Gracia, J.L.P.; Haanen, J. Evolving synergistic combinations of targeted immunotherapies to combat cancer. Nat. Rev. Cancer 2015, 15, 457-472. [CrossRef] [PubMed]

13. Cicero, A.F. Combination Therapy with Olmesartan/Hydrochlorothiazide to Improve Blood Pressure Control. J. Clin. Exp. Cardiol. 2015, 6, 384. [CrossRef]

14. Sun, W.; Sanderson, P.E.; Zheng, W. Drug combination therapy increases successful drug repositioning. Drug Discov. Today 2016, 21, 1189-1195. [CrossRef] [PubMed]

15. Terrie, Y.C. Monitoring combination drug therapy. Pharm. Times 2010, 76, 1-6.

16. Follath, F. Challenging the dogma of high target doses in the treatment of heart failure: Is more always better? Arch. Cardiovasc. Dis. 2009, 102, 785-789. [CrossRef] [PubMed]

17. Farr, M.; Bacon, P.A. How and when should combination therapy be used? The role of an anchor drug. Br. J. Rheumatol. (UK) 1995, 34, 100-103. [CrossRef]

18. Desai, D.; Wang, J.; Wen, H.; Li, X.; Timmins, P. Formulation design, challenges, and development considerations for fixed dose combination (FDC) of oral solid dosage forms. Pharm. Dev. Technol. 2013, 18, 1265-1276. [CrossRef]

19. Pourkavoos, N. Unique Risks, Benefits, and Challenges of Developing Drug-Drug Combination Products in a Pharmaceutical Industrial Setting. Comb. Prod. Ther. 2012, 2, 1-31. [CrossRef]

20. Goyanes, A.; Wang, J.; Buanz, A.; Martinez-Pacheco, R.; Telford, R.; Gaisford, S.; Basit, A.W. 3D printing of medicines: Engineering novel oral devices with unique design and drug release characteristics. Mol. Pharm. 2015, 12, 4077-4084. [CrossRef]

21. Khaled, S.A.; Burley, J.C.; Alexander, M.R.; Yang, J.; Roberts, C.J. 3D printing of five-in-one dose combination polypill with defined immediate and sustained release profiles. J. Control. Release 2015, 217, 308-314. [CrossRef] [PubMed]

22. Khaled, S.A.; Burley, J.C.; Alexander, M.R.; Yang, J.; Roberts, C.J. 3D printing of tablets containing multiple drugs with defined release profiles. Int. J. Pharm. 2015, 494, 643-650. [CrossRef] [PubMed]

23. Acosta-Vélez, G.F.; Linsley, C.S.; Craig, M.C.; Wu, B.M. Photocurable Bioink for the Inkjet 3D Pharming of Hydrophilic Drugs. Bioengineering 2017, 4, 11. [CrossRef] [PubMed]

24. Acosta-Vélez, G.F.; Zhu, T.Z.; Linsley, C.S.; Wu, B.M. Photocurable poly(ethylene glycol) as a bioink for the inkjet 3D pharming of hydrophobic drugs. Int. J. Pharm. 2018, 546, 145-153. [CrossRef] [PubMed]

25. Trachtman, H.; Frymoyer, A.; Lewandowski, A.; Greenbaum, L.A.; Feig, D.I.; Gipson, D.S.; Warady, B.A.; Goebel, J.W.; Schwartz, G.J.; Lewis, K.; et al. Pharmacokinetics, pharmacodynamics, and safety of lisinopril in pediatric kidney transplant patients: Implications for starting dose selection. Clin. Pharmacol. Ther. 2015, 98, 25-33. [CrossRef] [PubMed] 
26. Snauwaert, E.; Vande Walle, J.; De Bruyne, P. Therapeutic efficacy and safety of ACE inhibitors in the hypertensive paediatric population: A review. Arch. Dis. Child. 2017, 102, 63-71. [CrossRef] [PubMed]

27. Larik, F.A.; Saeed, A.; Shahzad, D.; Faisal, M.; El-Seedi, H.; Mehfooz, H.; Channar, P.A. Synthetic approaches towards the multi target drug spironolactone and its potent analogues/derivatives. Steroids 2017, 118, 76-92. [CrossRef] [PubMed]

28. United States Pharmacopoeia Convention. <1217> Tablet Breaking Force; USP 37-NF 32; United States Pharmacopeia and the National Formulary; The United States Pharmacopeial Convention: Rockville, MD, USA, 2014.

29. Jang, D.; Kim, D.; Moon, J.; Jang, D.; Kim, D.; Moon, J. Influence of Fluid Physical Properties on Ink-Jet Printability. Langmuir 2009, 25, 2629-2635. [CrossRef] [PubMed]

30. Cole-Parmer Instrument Company. Available online: https://pim-resources.coleparmer.com/ instructionmanual/surface-tension-apparatus-instruction-manual.pdf (accessed on 12 January 2017).

31. Department of Health and Human Services, Food and Drug Administration, Center for Drug Evaluation and Research (CDER). Size, Shape, and Other Physical Attributes of Generic Tablets and Capsules; Guidance for Industry; Center for Drug Evaluation and Research (CDER): Silver Spring, MD, USA, 2015.

32. Fraser, J.R.; Laurent, T.C.; Laurent, U.B. Hyaluronan: Its nature, distribution, functions and turnover. J. Intern. Med. 1997, 242, 27-33. [CrossRef] [PubMed]

33. Allison, D.D.; Grande-Allen, K.J. Review. Hyaluronan: A Powerful Tissue Engineering Tool. Tissue Eng. Part A 2006, 12, 2131-2140. [CrossRef] [PubMed]

34. Highley, C.B.; Prestwich, G.D.; Burdick, J.A. Recent advances in hyaluronic acid hydrogels for biomedical applications. Curr. Opin. Biotechnol. 2016, 40, 35-40. [CrossRef] [PubMed]

35. Owen, S.C.; Fisher, S.A.; Tam, R.Y.; Nimmo, C.M.; Shoichet, M.S. Hyaluronic acid click hydrogels emulate the extracellular matrix. Langmuir 2013, 29, 7393-7400. [CrossRef] [PubMed]

36. Kim, I.L.; Mauck, R.L.; Burdick, J.A. Hydrogel design for cartilage tissue engineering: A case study with hyaluronic acid. Biomaterials 2011, 32, 8771-8782. [CrossRef] [PubMed]

37. Juban, A.; Nouguier-Lehon, C.; Briancon, S.; Hoc, T.; Puel, F. Predictive model for tensile strength of pharmaceutical tablets based on local hardness measurements. Int. J. Pharm. 2015, 490, 438-445. [CrossRef] [PubMed]

38. Sheskey, P.J.; Cook, W.G.; Cable, C.G. Handbook Pharmaceutical Excipients, 6th ed.; Pharm Press: London, UK, 2009.

39. Williams, R.O.; Reynolds, T.D.; Cabelka, T.D.; Sykora, M.A.; Mahaguna, V. Investigation of excipient type and level on drug release from controlled release tablets containing HPMC. Pharm. Dev. Technol. 2002, 7, 181-193. [CrossRef] [PubMed]

40. Thomas, M.V.; Puleo, D.A. Calcium sulfate: Properties and clinical applications. J. Biomed. Mater. Res. Part B Appl. Biomater. 2009, 88, 597-610. [CrossRef] [PubMed]

41. Singh, N.B.; Middendorf, B. Calcium sulphate hemihydrate hydration leading to gypsum crystallization. Prog. Cryst. Growth Charact. Mater. 2007, 53, 57-77. [CrossRef]

42. Bešter-Rogač, M. Nonsteroidal Anti-Inflammatory Drugs Ion Mobility: A Conductometric Study of Salicylate, Naproxen, Diclofenac and Ibuprofen Dilute Aqueous Solutions. Acta Chim. Slov. 2009, 56, 70-77.

(C) 2018 by the authors. Licensee MDPI, Basel, Switzerland. This article is an open access article distributed under the terms and conditions of the Creative Commons Attribution (CC BY) license (http:/ / creativecommons.org/licenses/by/4.0/). 